\title{
$\widehat{A}$ Madridge \\ madridge Journal of Dentistry and Oral Surgery \\ Interconnecting Scientific World
}

Case Report

Open Access

\section{Oral Proliferative Verrucous Leukoplakia: The Unsolved Paradox}

\author{
Mervet Moussa $^{1,2 \star}$, Hend Salem ${ }^{1}$, and Sahar Mahmoud ElRefai ${ }^{3}$ \\ ${ }^{\prime}$ Professor of Oral Pathology, Faculty of Dentistry, Cairo University, Cairo, Egypt \\ ${ }^{2}$ Professor of Oral Pathology, College of Dentistry Princess Nourah University, Riyadh, KSA \\ ${ }^{3}$ Lecturer of Oral Pathology, Faculty of Dentistry, Fayoum University, Cairo, Egypt
}

Article Info

*Corresponding author:
Mervet Moussa
Professor
Faculty of Dentistry
Cairo University
Egypt
E-mail: drmervet@yahoo.com

Received: March 1, 2017

Accepted: March 27, 2017

Published: April 1, 2017

Citation: Moussa MM, Salem H, ElRefai SM. Oral Proliferative Verrucous Leukoplakia: The Unsolved Paradox. Madridge J Dent Oral Surg. 2017; 2(2): 55-58.

doi: $10.18689 / \mathrm{mjdl}-1000114$

Copyright: ( 92017 The Author(s). This work is licensed under a Creative Commons Attribution 4.0 International License, which permits unrestricted use, distribution, and reproduction in any medium, provided the original work is properly cited.

Published by Madridge Publishers

\begin{abstract}
Oral proliferative verrucous leukoplakia (OPVL) is a rare form of oral leukoplakia, which was first described in 1985 by Hansen et al, it is characterized by a distinct clinical form and defined by its progressive clinical course, changing clinical and histopathological features, and potential to develop into cancer usually higher than $70 \%$. OPVL is observed more frequently in elderly women, over 60 years at the time of diagnosis. The buccal mucosa and tongue are the most frequently involved sites. It develops initially as a white plaque of hyperkeratosis that eventually becomes a multifocal disease with confluent, exophytic and proliferative features with a progressive deterioration of the innocuous white oral lesions that it can resemble clinically. Characteristic histological features are lacking, and the diagnosis is based on the combination of clinical and histological findings showing progression of the lesion.
\end{abstract}

\section{Case Report}

We hereby report a case of 55 years old female patient having oral proliferative verrucous leukoplakia (OPVL), affecting different oral sites: lateral border of the tongue, gingival and buccal mucosae. Clinical intraoral examination revealed multifocal thick stiff white patches that could not be wiped off, differential diagnosis was made. Histological examination showed hyperkeratosis, acanthosis and dysplastic changes but there was not any frank invasion, a diagnosis of OPVL was made.

Keywords: Oral Cancer; Hyperkeratosis; Hyperplasia; White Lesions; Oral Proliferative Verrucous Leukoplakia.

\section{Introduction}

Head and neck squamous cell carcinoma (HNSSC) is a cancer of mucosal surface representing a major public health concern particularly oral cancer which is one of the most common neoplasms in developing countries. It is characterized by poor prognosis, low survival rate and constituting up to $25 \%$ of all types of cancer with a steady rise in its incidence over the last 20 years [1-3]. Oral cancer is usually preceded by potentially malignant disorders that could be presented as a white lesion. White lesions are relatively frequent in the oral cavity, oral leukoplakia being the most common presenting many clinical variants, one of which is oral proliferative verrucous leukoplakia (OPVL) a rare slowly growing type with long-term progression that could possibly turn malignant. It is recognized as a high risk non-homogenous subtype and observed more frequently in women and elderly patients at the time of diagnosis. 
The etiology of this condition is unknown and tobacco use does not seem to have a significant influence on the appearance or progression of (OPVL) and may occur both in smokers and nonsmokers. An association has been reported with human papillomavirus (HPV) especially HPV types 16 and 18, Epstein-Barr virus and Candidal infections $[4,5]$.

Though uncommon, OPVL is a distinctive lesion, reported by various published studies as a disease with an aggressive biological behavior, (precursor lesion of squamous cell carcinoma or verrucous carcinoma) and poor prognosis for this seemingly harmless-appearing white lesion of the oral mucosa (the rate of recurrence is high) therefore according to the latest World Health Organization nomenclature, OPVL conforms to the new terminology of "potentially malignant disorders [4-6]." The most noticeable clinical feature consists of a single homogeneous white patch, which progressively becomes multifocal exophytic, verrucous with erythematous areas. Although the entire oral mucosa may be involved, the most commonly affected areas are the tongue, gingiva, alveolar ridge, palatal and buccal mucosa [5-7].

Diagnosis is based on the correlation of clinical and microscopic findings showing progression of the lesion, for there are no characteristic histological features $[5,6]$.

The microscopic findings associated with OPVL are dependent on the stage of the disease and the adequacy of the biopsy. Hansen et al. [13] classified the pathological process of PVL into 10 grades, i.e, normal oral mucosa (0), homogeneous leukoplakia (2), verrucous hyperplasia (7), verrucous carcinoma (6), papillary squamous cell carcinoma (8), and poorly differentiated carcinoma (10), in which the odd scores refer to a status intermediate between those referred to by the adjacent even scores. Once PVL is confirmed, active therapy should be undertaken, such as surgery, laser management, photodynamic therapy, or combined treatments. However, OPVL responds poorly to various therapeutic measures, and its recurrence rate is relatively high, even after surgical removal [8].

\section{Case Report}

A 55 years old female was referred to the Maxillofacial Department College of Dentistry complaining of an intraoral white patch that she was not able to remove.

On taking history, the patient reported that it has been progressing for the last 6 months. She felt some rough area at the right side of the inner cheek region associated with inability to move her tongue laterally. She also stated that a very mild form of these lesions were present for 2 previous years and were diagnosed as lichen planus, and she was not under any treatment.

Clinical examination revealed a large, stiff white keratotic patch with erythematous areas on the left buccal mucosa adjacent to retro-molar areas White patches which were leathery and rough on palpation but non tender were present on the gingiva. A thick verrucous irregular, nonscrapable white lesion was seen on the lateral border of the tongue with an extended translucent thin patch on the floor of the mouth (Figures 1-4).
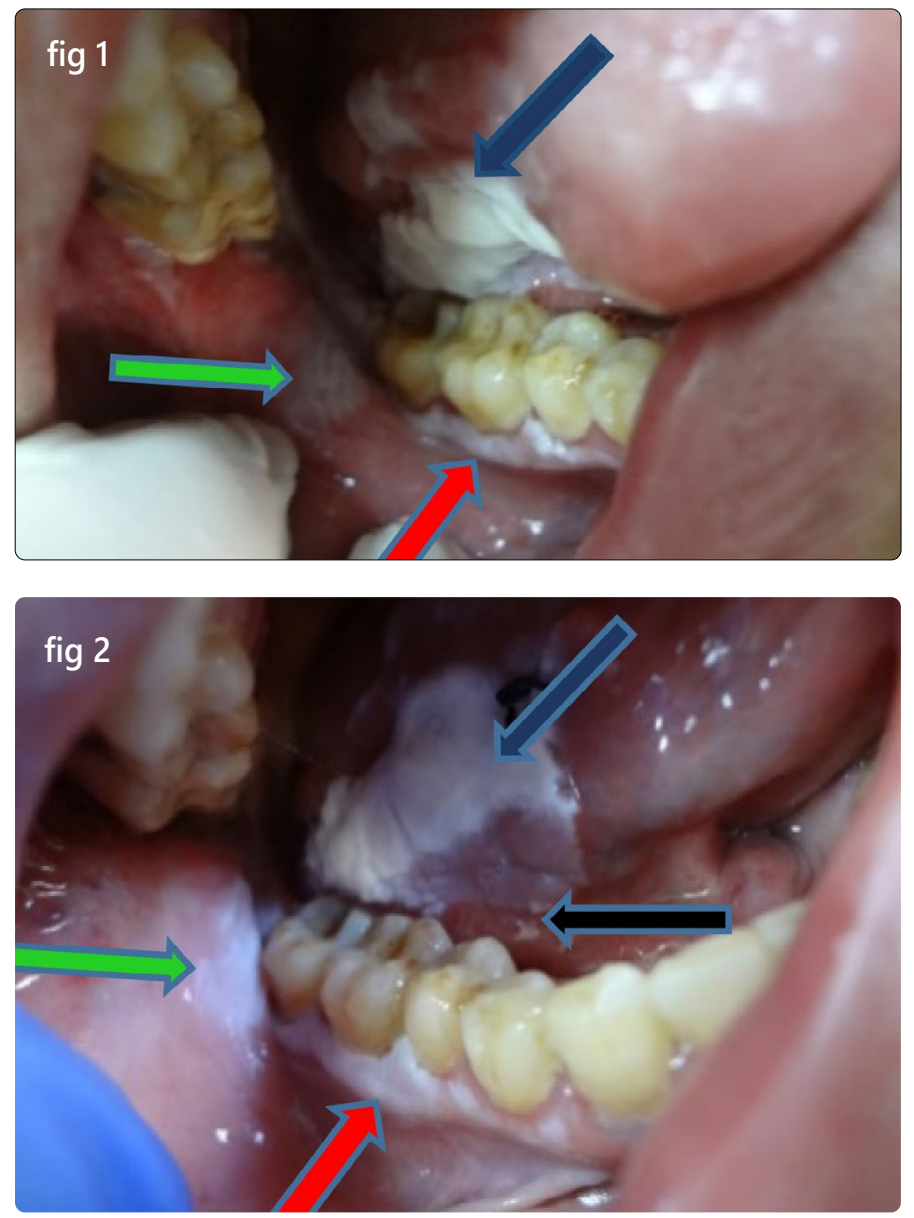

Figures 1 \& $\mathbf{2}$ showing a white verrucous lesion at the lateral border of the tongue (blue arrows), floor of the mouth (black arrow), gingival mucosa (red arrows), and buccal mucosa (green arrows).
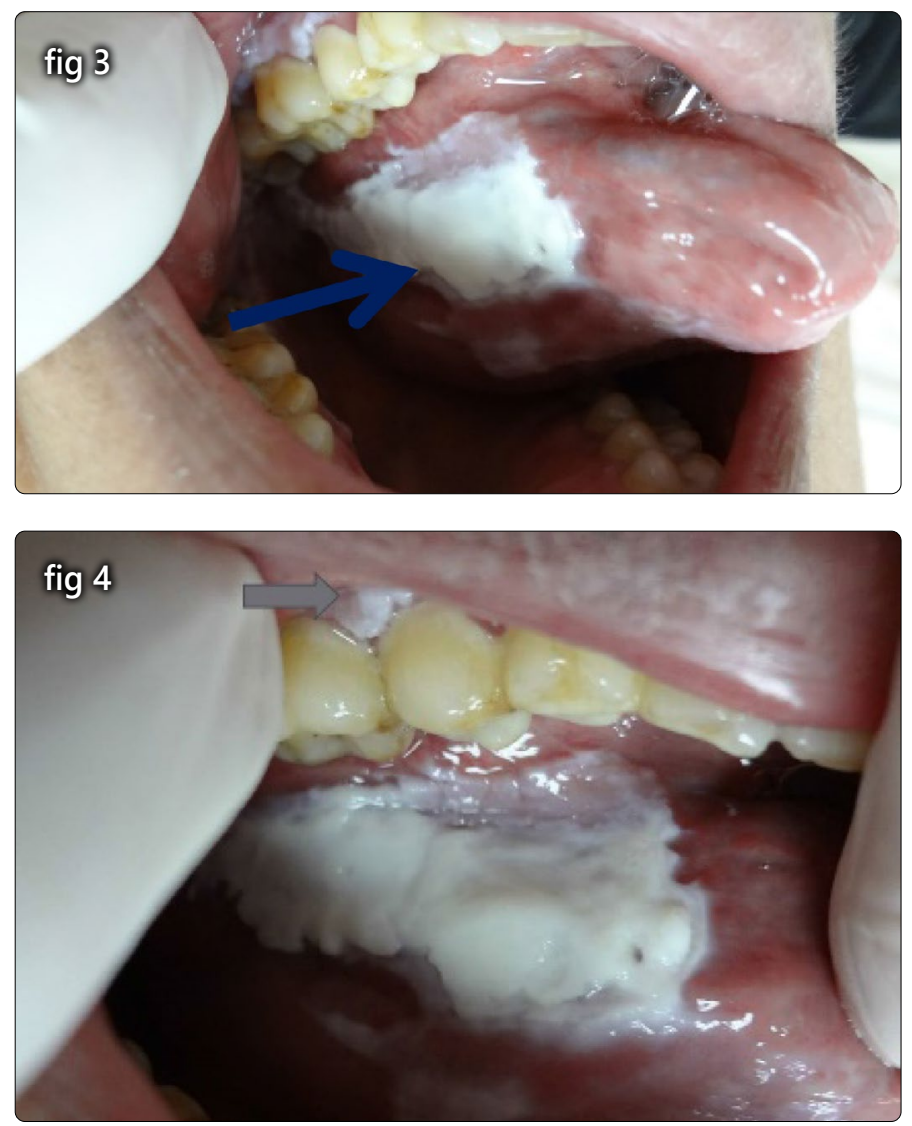

Figures $\mathbf{3} \boldsymbol{\&} \mathbf{4}$ showing a white verrucous lesion at the lateral border of the tongue (blue arrow). Note the thick white patch on maxillary gingival (grey arrow) 
Based on intraoral examination a differential diagnosis was made, including frictional keratosis, leukoplakia, verrucous carcinoma, PVL, Squamous cell carcinoma, Hyperplastic candidiasis, and lichen planus.

The treatment plan proceeded follows: 1). Patient was counseled about oral hygiene instructions. Phase 1) therapy (scaling) was performed. 2). A week later excisional biopsy of the lesion was done under local anaesthesia inclusive of the surrounding normal tissue and the patient was prescribed systemic antibiotics and antiseptic mouth wash.

\section{Histopathologic results}

Histopathologic examination of the biopsy revealed a stratified squamous epithelium which was hyperkeratotic in nature, whirling of epithelial cells with excessive keratin plug formation and keratin pearl were noted in the upper third of the epithelium. The underlying connective tissue was cellular, composed of dense chronic inflammatory infiltrate with few bundles of collagen (figures 5 and 6 ) both features reflect the thick white patch seen intraorally.

The rete ridges were bulbous / drop shaped, with basilar hyperplasia. Signs of dysplasia were evident in the epithelial layers particularly seen in the lower one third: loss of basal cells polarity with cell crowding, hyperchromatism, pleomorphism, increased nuclear cytoplasmic ratio, as shown in figure 7. Based on clinical and histopathological criteria, a diagnosis of OPVL was made.

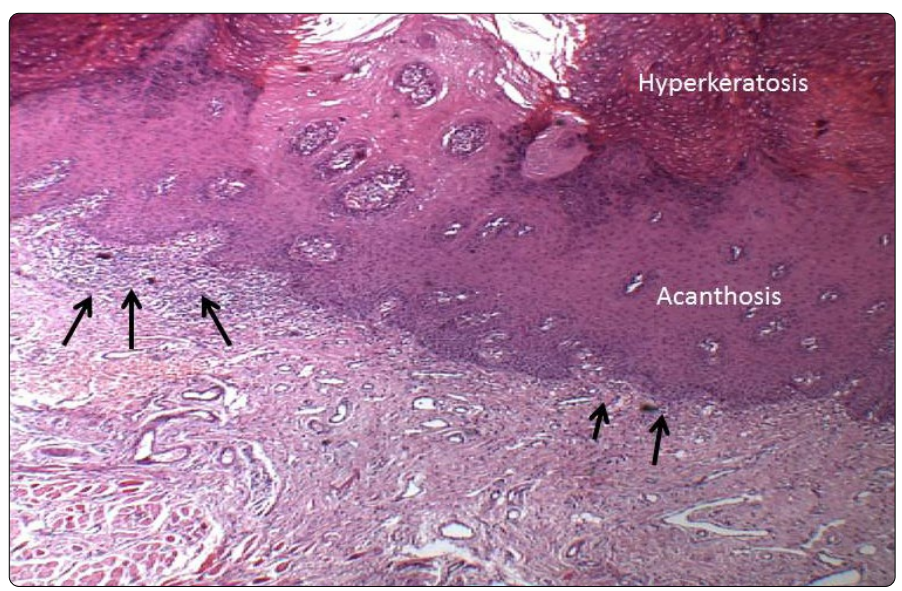

Figure 5: Showing stratified squamous epithelium with excessive hyperkeratosis and acanthosis (H\&Ex10) and the chronic inflammatory cells infiltration just beneath the epithelium in the underlying connective tissue. (Black arrows) (H\&Ex40).

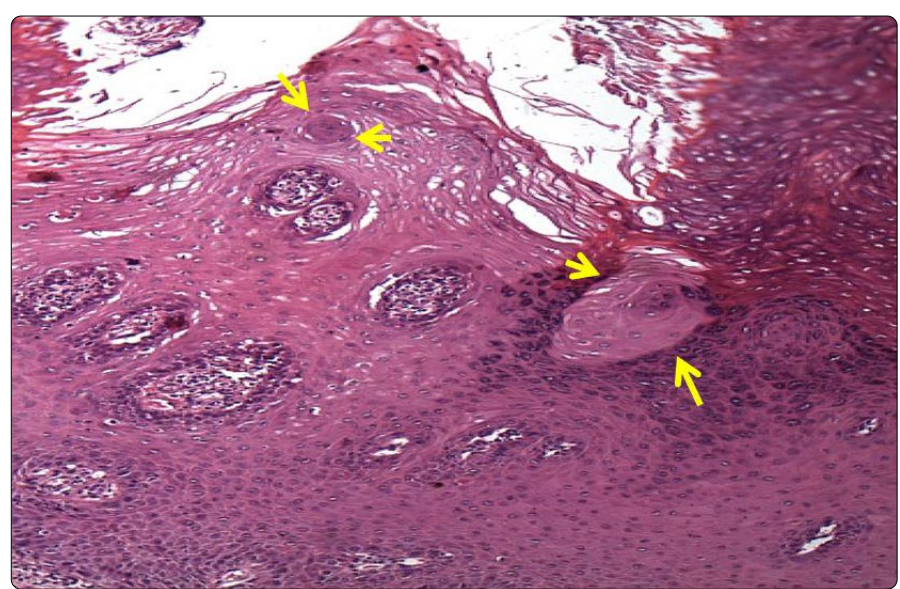

Figure 6: Note the keratin pearl formation in the prickle cell layer (yellow arrows)

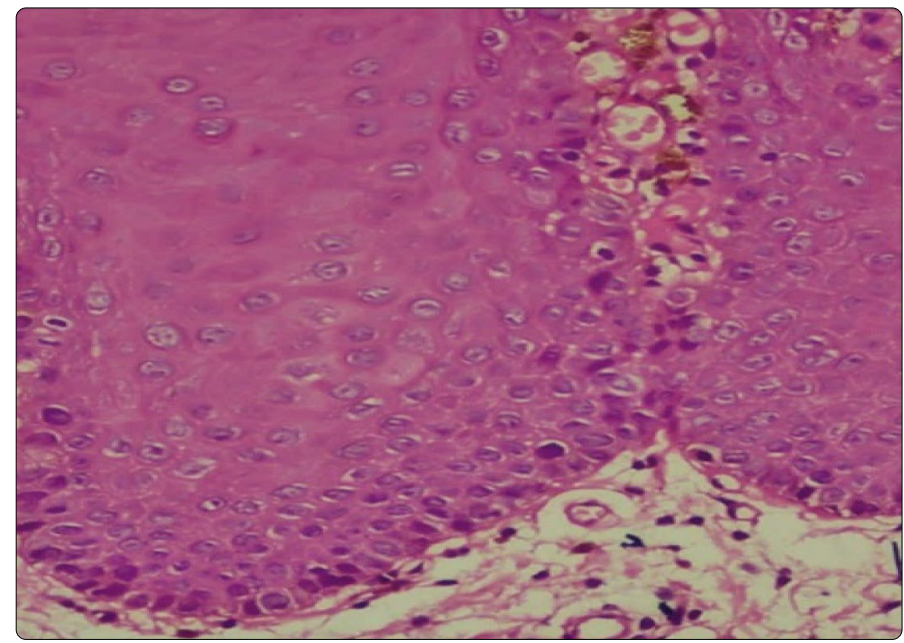

Figure 7: Note the broad rete ridges, signs of mild epithelial dysplasia (H\&Ex40)

\section{Discussion}

White lesions are relatively frequent in the oral cavity, leukoplakia is by far the most common oral potentially malignant disorder representing $85 \%$ of such lesions. OPVL is a progressive condition which develops initially as a white plaque of hyperkeratosis and eventually becomes a multifocal disease with proliferative and papillary features. The etiology of this condition has been very puzzling for years and little has been proved about its origin. Suggestions included expression of transforming growth factor-alpha, DNA ploidy, Epstein Barr virus, p53 expression and human papilloma virus, but none was satisfactory [9-11].

For many years, case reports identified characteristic predisposition in women (4:1) and older patients (60 years mean age), with the buccal mucosa and tongue as the most commonly affected sites. The gingiva is also commonly affected and hence the term Proliferative Verrucous Leukoplakia of the Gingiva (PVLG) was introduced [12].

Most reported data showed that the lesion is irreversible, may include red areas or may manifest as a red rather than a white lesion, and is resistant to all forms of treatment with a high recurrence rate $[13,14]$.

Hansen et al. suggested a histopathological view for this lesion which was later modified by Batsakis et al, which included: simple hyperkeratosis, which can progress, with or without stages of epithelial dysplasia, to verrucous hyperplasia, verrucous carcinoma or squamous cell carcinoma [14].

Morton et al noticed in their histopathologic samples, cells showing both basal cell degeneration and lymphocytic infiltration just beneath the epithelium, pointing out that early stages of OPVL may be mistaken for oral lichen planus or (lichenoid pattern) [15].

In 2010 Cerero-Lapiedra et al. [16] have proposed major and minor histopathological criteria for the diagnosis of (OPVL). More recently, however Carrad et al. [14] stated that the major criteria should not include keratinized mucosa as the most frequently affected site but should also consider any site of the oral mucosa [11]. In the present case, the biopsy taken revealed stratified squamous epithelium showing hyperkeratosis with 
corrugated keratin (Figures 5 and 6) hyperplasia and signs of mild epithelial dysplasia (Figure 7). It should be noted that in cases of multifocality, while one lesion may be simple hyperkeratosis, another one located elsewhere may already have developed into a verrucous hyperplasia, verrucous carcinoma, oral squamous carcinoma whether in situ or infiltrating, as described by Hansen stating that (OPVL) develops through a histopathological continuum not all lesions are at the same stage of evolution [16]. Chronic inflammatory cell infiltration of the underlying connective tissue was also evident.

Consequently, the present study satisfies and confirms the major and minor criteria set by Cerero-Lapiedraet al and Carrard et al; as it involved more than two different clinical sites(major criteria) and histopathological examination showed hyperkeratosis, hyperplasia and epithelial dysplatic features which fulfill the minor criteria.

No consent form was used as patients coming to the Maxillofacial Surgery Department were considered as educational cases, and his or her information was used for research purpose. The patient in the present case was aware with this information and our main concern here was the histopathological changes occurring in this enigmatic (OPVL) case.

\section{Conclusion}

In conclusion, the present case was diagnosed as OPVL, one of the aggressive white lesions affecting different oral locations. Palliative treatment was carried out, as the patient disapproved when surgery, laser and radiotherapy were proposed.

Proliferative verrucous leukoplakia is known for its progressive behavior and tendency to recur after its removal as no specific treatment modality has proven to be effective in aborting its progression. Additionally because of its high recurrence potential and relentless progression to squamous cell carcinoma, all recurrent and multifocal white lesions of the oral cavity should be viewed with suspicion.

\section{References}

1. Vitale-Cross L, Molinolo A, Martin D, et al. Metformin Prevents the Development of Oral Squamous Cell Carcinomas from CarcinogenInduced Premalignant Lesions. Cancer Prev Res. 2012; 5: 562-73. doi: 10.1158/1940-6207.CAPR-11-0502
2. Kitakawaa D, Cabrala L, Marquesb M, Salvadorib D, Ribeiro D. Mediumterm tongue carcinogenesis assays: A comparative study between nitroquinolone oxide 1(4NQO)-induced rat and dimethyl-benzanthracene (DMBA)-induced hamster carcinogenesis. Journal of Experimental Animal Science. 2006; 43: 219-227.

3. Majchrzak E, Szybiak B, Wegner A, et al. Oral cavity and oropharyngeal squamous cell carcinoma in young adults: a review of the literature. Radiol Oncol. 2014; 48: 1-10. doi: 10.2478/raon-2013-0057

4. Issrani R, Prabhu N, Keluskar V. Oral proliferative verrucous leukoplakia: A case report with an update. Contemp Clin Dent. 2013; 4(2): 258-262. doi: 10.4103/0976-237X.114887

5. García-Delaney C, Vidal-Bel A, Sánchez-Garcés MÁ, Gay-Escoda C. Proliferative verrucous leukoplakia: a case report with characteristics of long-term progression. Oral Surg. 2016; 9: 243-247. doi: 10.1111/ ors. 12203

6. Bruch JM, Treister NS. Clinical oral medicine and pathology. New York. Humana Press. 2010; 121-122. doi: 10.1007/978-1-60327-520-0

7. Gandolfo S, Castellani R, Pentenero M. Proliferative verrucous leukoplakia: a potentially malignant disorder involving periodontal sites. J Periodontol. 2009; 80: 274-81.

8. Ge L, Wu Y, Wu LY, et al. Case report of rapidly progressive proliferative verrucous leukoplakia and a proposal for etiology in mainland China. World Journal of Surgical Oncology. 2011; 9: 26. doi: 10.1186/14777819-9-26

9. Neville BW, Damm DD, Allen CM, Bouquot J. Oral and maxillofacial pathology, 3rd edition. Saunders. pp 388-97,422-3.

10. Bagan J, Scully $C$, Jimenez $Y$, Martorell M. Proliferative verrucous leukoplakia: a concise update. Oral Diseases. 2010; 16: 328-32. doi: 10.1111/j.1601-0825.2009.01632.x

11. Van der Waal I, Reichart PA. Oral proliferative verrucous leukoplakia revisited. Oral Oncol. 2008; 44(8): 719-21. doi: 10.1016/j.oraloncology.2007.09.010

12. Gillenwater $A M$, Vigneswaran $N$, Fatani $H$, Saintigny $P$, El-Naggar AK Proliferative verrucous leukoplakia (PVL): recognition and differentiation from conventional leukoplakia and mimics. Head Neck. 2013. doi: 10.1002/hed.23505.

13. Hansen LS, Olson JA, Silverman S Jr. Proliferative verrucous leukoplakia. A long-term study of thirty patients. Oral Surg Oral Med Oral Pathol. 1985; 60(3): 285-98.

14. Carrard VC, Brouns ER, van der Waal I. Proliferative verrucous leukoplakia; a critical appraisal of the diagnostic criteria. Med Oral Patol Oral Cir Bucal. 2013; 18(3): 411-3. doi: 10.4317/medoral.18912

15. Morton TH, Cabay RJ, Epstein JB. Proliferative verrucous leukoplakia and its progression to oral carcinoma: report of three cases. J Oral Pathol Med. 2007; 36(5): 315-8. doi: 10.1111/j.1600-0714.2007.00499.x

16. Cerero-Lapiedra R, Baladé-Martínez D, MorenoLópez LA, EsparzaGómez G, BagánJV. Proliferative verrucous leukoplakia: a proposal for diagnostic criteria. Med Oral Pathol Oral Cir Bucal. 2010; 15(6): 839-45. doi: $10.4317 /$ medoral.15.e839 